\title{
Beier, Frank
}

\section{Soziologische Methoden und erziehungswissenschaftliche Theorie? Ein kritischer Diskussionsbeitrag zum Theorie-Empirie-Problem der qualitativen Forschung in der Pädagogik}

Erziehungswissenschaft 29 (2018) 56, S. 65-75

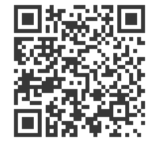

Quellenangabe/ Reference:

Beier, Frank: Soziologische Methoden und erziehungswissenschaftliche Theorie? Ein kritischer Diskussionsbeitrag zum Theorie-Empirie-Problem der qualitativen Forschung in der Pädagogik - In: Erziehungswissenschaft 29 (2018) 56, S. 65-75 - URN: urn:nbn:de:0111-pedocs-157184 - DOI: 10.25656/01:15718

https://nbn-resolving.org/urn:nbn:de:0111-pedocs-157184 https://doi.org/10.25656/01:15718

in Kooperation mit / in cooperation with:

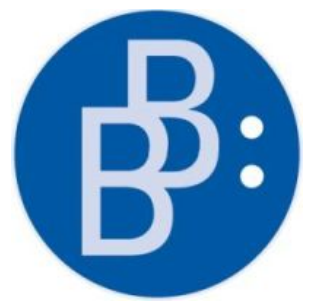

https://www.budrich.de

\section{Nutzungsbedingungen}

Dieses Dokument steht unter folgender Creative Commons-Lizenz: http://creativecommons.org/licenses/by-nd/4.0/deed.de - Sie dürfen das Werk bzw, den Inhalt vervielfältigen, verbreiten und öffentlich zugänglich machen, solange Sie den Namen des Autors/Rechteinhabers in der von inm festgelegten Weise nennen und das Werk bzw. diesen Inhalt nicht bearbeiten, abwandeln oder in anderer Weise verändern.

Mit der Verwendung dieses Dokuments erkennen Sie die Nutzungsbedingungen an.

\section{Terms of use}

This document is published under following Creative Commons-License: http://creativecommons.org/licenses/by-nd/4.0/deed.en - You may copy, http://creativecommons.org/licenses/by-nd/4.0/deed.en - You may copy, distribute and transmit, adapt or exhibit the work in the public as long as you
attribute the work in the manner specified by the author or licensor. You are attribute the work in the manner specified by the author or licensor. You are not allowed to alter or transform this work or its contents at all.

By using this particular document, you accept the above-stated conditions of use.

\section{(c) $(\ominus)$}

\section{Kontakt / Contact:}

\section{peDOCS}

DIPF | Leibniz-Institut für Bildungsforschung und Bildungsinformation Informationszentrum (IZ) Bildung

E-Mail:pedocs@dipf.de

Internet: www.pedocs.de

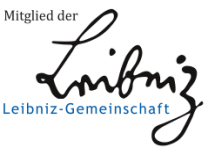




\section{Erziehungswissenschaft}

Mitteilungen der Deutschen Gesellschaft für Erziehungswissenschaft

\section{Heft 56, Jg. 29|2018}

\section{Der Beitrag der Erziehungswissenschaft zur Bildungsforschung}

Mit Beiträgen von

Elmar Anhalt, Frank Beier, Dietrich Benner, Ulrich Binder, Rita Casale, Johannes Drerup, Maria Hallitzky, Franziska Heinze, Christian Herfter, Sascha Kabel, Daniel Kasper, Robert Kreitz, Gabriele Molzberger, Marion Pollmanns, Sabine Reh, Thomas Rucker, Joachim Scholz, Michael Schurig, Karla Spendrin, Felicitas Thiel, Christiane Thompson, Gaudenz Welti 


\section{INHALTSVERZEICHNIS}

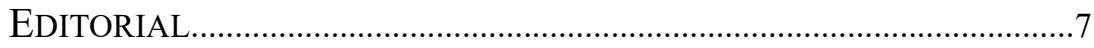

\section{THEMENSCHWERPUNKT}

„DER BEITRAG DER ERZIEHUNGSWISSENSCHAFT ZUR

BILDUNGSFORSCHUNG“6

Dietrich Benner

Der Beitrag der Erziehungswissenschaft zur Bildungsforschung, erörtert aus der Perspektive der Allgemeinen Erziehungswissenschaft und der Erziehungs- und Bildungsphilosophie

Elmar Anhalt, Thomas Rucker \& Gaudenz Welti

Erziehung als Ermöglichung von Bildung. Über die originäre

Problemstellung der Erziehungswissenschaft im Kontext der

Bildungsforschung

Johannes Drerup

Bildungsforschung: Beiträge der Erziehungs- und Bildungsphilosophie......27

Felicitas Thiel

Der Beitrag der empirischen Erziehungswissenschaft zur empirischen

Bildungsforschung

Michael Schurig \& Daniel Kasper

Zur Interpretation von Messfehlern aus Sicht der

Erziehungswissenschaft

Robert Kreitz

Das Erziehungswissenschaftliche der qualitativen Bildungsforschung

Frank Beier

Soziologische Methoden und erziehungswissenschaftliche Theorie?

Ein kritischer Diskussionsbeitrag zum Theorie-Empirie-Problem der qualitativen Forschung in der Pädagogik....

Sascha Kabel \& Marion Pollmanns

Wider die Diffusion des Bildungsbegriffs. Über die erziehungswissenschaftliche Notwendigkeit, die Reproduktion von Bildungsungleichheit durch pädagogische Rekonstruktion von Unterricht $\mathrm{zu}$ verstehen 
Maria Hallitzky, Franziska Heinze, Christian Herfter \& Karla Spendrin Potenziale der normativen Valenz allgemeindidaktischer Theorien für die Bildungsforschung .....

Ulrich Binder

Die erziehungswissenschaftliche Programmstruktur: Operationen im

Forschungsreich der Mitte

Christiane Thompson

Umstrittene Gründe. Erziehungswissenschaftliche Beiträge zur Bildung und Kritik des Wissens der Bildungsforschung

Sabine Reh \& Joachim Scholz

Historische Bildungsforschung und ihre erziehungswissenschaftlichen Perspektive.

\section{ALLGEMEINE BEITRÄGE}

Rita Casale \& Gabriele Molzberger

Studium Generale in der BRD nach 1945. Zu Konstitution und Wandel universitärer Bildungsformate.

\section{LAUDATIONES}

Hans-Christoph Koller

Laudatio für Prof. Dr. Werner Helsper anlässlich der Verleihung des Ernst-Christian-Trapp-Preises.

Hannelore Faulstich-Wieland, Ingrid Miethe \& Hans-Rüdiger Müller

Laudatio für Prof. Dr. Michael Göhlich anlässlich der Verleihung des Forschungspreises der DGfE

Hannelore Faulstich-Wieland, Ingrid Miethe \& Hans-Rüdiger Müller

Laudatio für Prof. Dr. Susanne Maria Weber anlässlich der Verleihung des Forschungspreises der DGfE

Hannelore Faulstich-Wieland, Ingrid Miethe \& Hans-Rüdiger Müller

Laudatio für Prof. Dr. Andreas Schröer anlässlich der Verleihung des

Forschungspreises der DGfE

Natalie Fischer

Laudatio für Dr. Frederick de Moll anlässlich der Verleihung des

Förderpreises der DGfE 


\section{Malte Brinkmann}

Laudatio für Dr. Christian Nerowski anlässlich der Verleihung des

Förderpreises der DGfE

Petra Bauer

Laudatio für PD Dr. Anke Wischmann anlässlich der Verleihung des

Förderpreises der DGfE

Hermann Josef Abs

Laudatio für Prof. Dr. Klaus Beck anlässlich der Verleihung der

Ehrenmitgliedschaft der DGfE.

Hans-Christoph Koller

Laudatio für Prof. Dr. Margret Kraul anlässlich der Verleihung der

Ehrenmitgliedschaft der DGfE

Burkhard Schäffer

Laudatio für Prof. Dr. Rudolf Tippelt anlässlich der Verleihung der

Ehrenmitgliedschaft der DGfE.

\section{MitTEILUNGEN DES VORSTANDS}

Rechenschaftsbericht

über die Vorstandsperiode März 2016 bis März 2018

Einladung zur Mitgliederversammlung der DGfE

am 20. März 2018 in Essen

Tagesordnung der Mitgliederversammlung der DGfE.

Antrag auf Änderung der DGfE-Beitragsordnung für die

Mitgliederversammlung am 20. März 2018 in Essen

Bericht der Programmkommission über die Beitragsauswahl

für den DGfE-Kongress „Bewegungen“ in Essen 2018.

Empfehlungen guter Arbeits- und Beschäftigungsbedingungen von

Wissenschaftler*innen in Qualifikationsphasen in der

Erziehungswissenschaft

Anna Moldenhauer, Christian Nerowski \& Doris Wittek

Der Unterschied von Leitbild und Abbild. Eine Stellungnahme. 186 
Call for Posters zum DGfE-Workshop

„Die aktuelle Lage der Methodenausbildung in Erziehungswissenschaft und Bildungsforschung“"

\section{BERICHTE AUS DEN SEKTIONEN}

Sektion 1 - Historische Bildungsforschung

Sektion 2 - Allgemeine Erziehungswissenschaft.... 194

Sektion 3 - Interkulturelle und International Vergleichende Erziehungswissenschaft (SIIVE) 201

Sektion 5 - Schulpädagogik .204

Sektion 8 - Sozialpädagogik und Pädagogik der frühen Kindheit...............206

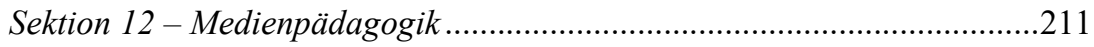

Sektion 13 - Differentielle Erziehungs- und Bildungsforschung .................213

Sektion 14 - Organisationspädagogik ....................................................216

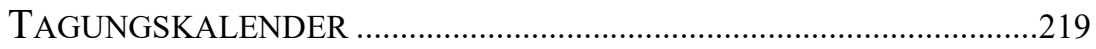




\section{Soziologische Methoden und erziehungswissenschaftliche Theorie?}

\section{Ein kritischer Diskussionsbeitrag zum Theorie-Empirie-Problem der qualitativen Forschung in der Pädagogik}

\section{Frank Beier}

\section{Einleitung: Erziehungswissenschaft und ihre Empirie}

Ob und wie die Erziehungswissenschaft selbst empirisch forschen soll, ist ein seit Dekaden diskutiertes Problem dieser Disziplin. Dies lässt sich exemplarisch an der historischen Entwicklung der institutionellen Verankerung der empirischen Bildungsforschung skizzieren: Während ab den 1960er Jahren im Zuge der von Heinrich Roth proklamierten „realistischen Wendung“ (Roth 1962) vorrangig die außeruniversitäre empirische Bildungsforschung anwuchs, kam es seit dem sogenannten PISA-Schock Anfang der 2000er Jahre auch an den Universitäten zu einer flächendeckenden Expansion von Lehrstühlen für empirische Bildungsforschung (vgl. Zedler/Döbert 2009, S. 33). Dieser in den letzten Jahren bildungspolitisch protegierte Aufstieg der (vorwiegend quantitativen) Bildungsforschung und die Skepsis der traditionellen Pädagogik gegenüber empirischer Forschung (vgl. Dinkelaker et al. 2016) begünstigten jedoch, dass vor allem die beiden Nachbardisziplinen Soziologie und Psychologie sich Forschungsfelder aufteilten und ihre Methodenexpertise einbringen konnten. Der Erziehungswissenschaft mit ihren Theoriebeständen und Grundbegrifflichkeiten wurde und wird dabei kaum mehr eine größere Rolle innerhalb der Bildungsforschung zugewiesen. So resümiert Cornelia Gräsel beispielsweise:

„Insgesamt kann aufgrund der theoretischen, methodischen und normativen Unterschiede - man vergleiche die lang anhaltende Debatte um die unterschiedlichen Bildungsbegriffe - nicht sinnvoll von einer Empirischen Bildungsforschung als Teildisziplin der Erziehungswissenschaft gesprochen werden.“ (Gräsel 2015, S. 27)

Während kein Zweifel besteht, dass die Bildungsforschung im Kern ein interdisziplinäres Feld ist, bleibt hingegen die Frage, welche Rolle die Erziehungswissenschaft selbst in der interdisziplinären Kooperation spielen kann, mehr oder minder umstritten. Ich möchte mich in diesem Aufsatz speziell auf die qualitative Bildungsforschung beschränken, die neben der deutlich dominanteren quantitativen Bildungsforschung spätestens seit den 1990er Jahren in der Erziehungswissenschaft an Bedeutung zu gewinnen scheint, und diskutieren, inwieweit eine eigenständig erziehungswissenschaftliche Perspektive 
entwickelt wurde, die sich von impliziten soziologischen und psychologischen Gegenstandskonstitutionen abzugrenzen vermag.

\section{Erziehungswissenschaft als Sozialwissenschaft}

Es gibt eine Reihe von Autorinnen und Autoren, die qualitative Auswertungsverfahren aus der Soziologie in die Erziehungswissenschaft übernommen haben. So wird in der qualitativen Unterrichtsforschung beispielsweise die dokumentarische Methode (vgl. z.B. Asbrand/Martens/Petersens 2013) oder die Konversationsanalyse verwandt (vgl. z. B. Wyßuwa/Beier 2012) oder im Anschluss an die Tradition der Chicagoer Schule und der Ethnomethodologie eine Ethnografie des Unterrichts angefertigt (vgl. z.B. Breidenstein 2012). Zwar reagieren die genannten Studien auf pädagogische Konzepte und erziehungswissenschaftliche Begriffe, treten aber quasi von außen heran und betreiben empirische Aufklärung über diese. Das ,Erziehungswissenschaftliche" oder das ,Pädagogische' solcher Art Studien besteht folglich im Gegenstand, der adressiert wird. Nach ihrem Selbstverständnis sind diese Studien erziehungswissenschaftlich, weil sie bestimmte Themen wie Unterricht, Erziehung oder Bildung behandeln. Merle Hummrich und Astrid Hebenstreit haben diese Position kürzlich explizit verteidigt:

„Insofern konstituiert sich Erziehungswissenschaft vornehmlich über ihren Gegenstand, als eine Wissenschaft, die sich mit Perspektiven der Bildung, Erziehung und Sozialisation auseinandersetzt, die Situationen, Institutionen und kulturelle Bedingungen vergleicht und zueinander in Beziehung setzt." (Hummrich/Hebenstreit 2017, S. 3)

Solche Gegenstände können zum Beispiel der individualisierte Unterricht (vgl. Breidenstein/Menzel/Rademacher 2013), die Behandlung spezifischer Themen im Unterricht (am Beispiel Holocaust, Meseth 2008) oder wie im oben zitierten Vortrag von Hummrich und Haberstedt (2017) die Herstellung von Disziplin im Rahmen einer Schulkultur sein. Diese qualitativen Studien haben wichtige Erkenntnisse zur Explikation der sozialen Praxis von Bildungs- und Erziehungsprozessen geliefert. Die Anwendung der klassischen qualitativen Auswertungsverfahren bietet eine attraktive Möglichkeit, sich von idealistischen und normativ aufgeladenen Begriffen zu lösen, mit denen man in pädagogischen Theorien häufig konfrontiert ist. Es dürfe gerade nicht - so das häufig vorgetragene Credo - eine Vermischung mit den normativen Erwartungen, die den Gegenstand berühren und den eingesetzten Forschungsmethoden geben. So argumentiert etwa Peter Vogel:

„Im empirischen Zugriff ist die ,Eigennormativität‘ des Pädagogischen zwar notwendiges Element der pädagogischen Handlungstheorie, aber einer Handlungstheorie als Gegenstand von Beobachtung.“ (Vogel 2016, S. 230) 
Der erziehungswissenschaftlichen Forschung wird somit aufgetragen, pädagogische Normen des Feldes zu rekonstruieren, ohne diese dabei durch eigene Forschung zu reifizieren. Peter Vogel steht in der Tradition Wolfgang Brezinkas, der gut 40 Jahre zuvor die Versozialwissenschaftlichung der Pädagogik forderte. Interessanterweise ging Brezinka deutlich weiter und bestritt auch, dass die Erziehungswissenschaft einen ihr eigenen Gegenstand besitze:

„Die Behauptung, die Pädagogik habe einen Gegenstand, der von den Gegenständen der ,Nachbarwissenschaften' Psychologie und Soziologie gänzlich verschieden sei, läßt sich ebenso wenig halten wie der Anspruch auf eine nur ihr eigentümliche Methode. Soweit diese Ansichten heute noch vertreten werden, beziehen sie sich zumeist nicht auf die Pädagogik als Realwissenschaft, sondern als eine philosophische Disziplin.“ (Brezinka 1971, S. 37)

Die Aufgabe der Erziehungswissenschaft sah Brezinka darin, unter Zuhilfenahme sozialwissenschaftlichen Wissens, Erziehungstechnologien zu entwerfen. Dieser Technologieanspruch wird mittlerweile insbesondere in der qualitativen Forschung abgestritten. Funktional-äquivalent wird stattdessen erziehungswissenschaftliche Reflexion gefordert, die das Wesen der qualitativen Forschung in der Erziehungswissenschaft ausmache und zu gleich konstitutiv für eine pädagogische Kasuistik angesehen wird. ${ }^{1}$ Qualitative Methoden, die zur Analyse von Unterricht oder anderen Elementen des Bildungssystems genutzt werden, gehören dann quasi automatisch zur Erziehungswissenschaft, allein deshalb, weil sie „Unterricht" thematisieren und zu dessen Reflexion beitragen können. Tendenziell ausgeblendet wird damit jedoch, dass Methoden ihren Gegenstand nicht einfach aufgreifen, sondern ihn immer durch die mit ihr verbundene aspekthafte Betrachtung zugleich auch konstituieren:

„Forschung ,schafft‘ somit ihren Gegenstand (in gewisser Weise) und damit auch die zu erhebenden Daten - weder das Eine noch das Andere findet sie einfach in der AuBenwelt vor (Breuer 2005). Deshalb sind Methoden immer theoretisch geleitet und deshalb sind die ausgearbeiteten ,Methoden' immer auch Theorie: Gesellschaftstheorie, Sozialtheorie, Handlungstheorie, Kommunikations- und Medientheorie und Erkenntnistheorie in einem." (Reichertz 2012, S. 72)

Diese Gegenstandskonstitution ist allen Forschungsmethoden immanent. Auch qualitative Verfahren verweisen auf eine „Theorie des Gegenstandes, genauer: einer Grundlagentheorie der Soziologie“ (Knoblauch 2015, S. 215). Für Gesa Lindemann liefern Sozialtheorien in der qualitativen Forschung den Rahmen, „wie der Gegenstand zu beobachten ist und empirische Daten zu interpretieren

1 Dies wird zum Beispiel im Rahmen der reflexiven Lehrerbildung diskutiert (vgl. Berndt/Häcker/Leonhard 2017). Werner Helsper (2001, S. 12) spricht in diesem Zuge von der Ausbildung eines ,wissenschaftlich-reflexiven Habitus“, Bettina Dausien (2007) sieht in dem Erlernen qualitativer Forschung die Möglichkeit, Professionalisierung voranzutreiben. 
sind“ und ,[...] wie etwas als soziologisches empirisches Datum erscheinen kann" (Lindemann 2015, S. 109). Nimmt man diese immanenten Gegenstandskonstitutionen ernst, so bedeutet dies, dass auch Erziehung, Bildung und Sozialisation durch die klassischen qualitativen Methoden zunächst einmal als soziologische Konstrukte konstituiert werden. In der Tat erscheint es ja sofort einsichtig, dass der ,gleiche“ (aber nicht-identische) Gegenstand, z. B. Erziehung, unter ganz unterschiedlichen disziplinären Perspektiven erforscht werden kann: Es macht freilich einen Unterschied, ob ich Erziehung als phylogenetische Überlebensstrategie für physiologische Frühgeburten untersuche, als psychodynamische Übertragung und Gegenübertragung oder als kontingentes Konstrukt sozialer Reproduktion. Um entsprechende Fakten für die jeweiligen Perspektiven zu sammeln, benötige ich entsprechende andere empirische Verfahren und Instrumente. Es ist also keineswegs so, dass Erziehung allein als soziale Praxis untersucht werden kann, auch wenn dies die klassischen soziologischen Analyseverfahren nahelegen. Dies wird jedoch häufig suggeriert. Peter Vogel beispielsweise hält die Vorstellung für abwegig, dass eine pädagogische Forschung eine spezifische bildungstheoretisch fundierte Gegenstandskonstitution benötigt, und illustriert dies mit der folgenden Analogie:

„Wir fänden es ziemlich eigenartig, wenn in der Religionssoziologie eine empirische Untersuchung über die Bedeutung religiöser Motive im Alltagshandeln von Kirchgängern mit dem Argument kritisiert würde, man habe ja gar nicht die richtige und allein wahre Religion untersucht. Vergleichbare Argumente finden sich aber reichlich im Zusammenhang der Kritik der empirischen Bildungsforschung." (Vogel 2016, S. 230)

Gleichermaßen wie die Erforschung von Religion keine religiöse Perspektive brauche, sei es unsinnig, Bildungsforschung zu kritisieren, weil sie nicht bildungstheoretisch aufgestellt sei. Es ist Peter Vogel zunächst einmal vollumfänglich zuzustimmen: Wer etwas über Religion herausfinden will, der kann Religion als soziale Praxis oder soziales Phänomen untersuchen. Dies ist aber nichts anderes als eine theoretische Gegenstandskonstitution - Religion wird als soziales und nicht etwa als sozialpsychologisches, juristisches oder ästhetisches, philosophisches (oder eben religiöses) Phänomen betrachtet resp. konstituiert. Die Konsequenz aus Vogels Überlegung ist, dass die Erforschung von Religion hier zwangsläufig zu einer Religionssoziologie wird. Denn es ist das Kerngeschäft der Soziologie, die Logik sozialer Praxis zu rekonstruieren. Eine theologische Untersuchung andererseits benötigte genau jene Spezifizierung des Religiösen (wieso ist ein Gegenstand heilig/religiös?), die den Gesichtskreis der Forschung leiten würde, deren wissenschaftliche Berechtigung Vogel für eine Theologie m. E. zu Recht abstreitet. Lautet die Konsequenz daraus, dass eine pädagogische Perspektive (was macht etwas zu etwas Pädagogischem?) gleichermaßen zum Scheitern verurteilt ist? Gerade dies scheint Vogels Vergleich insinuieren zu wollen. Wenn dem so wäre, so bliebe nur die Konsequenz, Erziehungswissenschaft als Erziehungs- 
soziologie fortzuschreiben und pädagogische Handlungen als eine spezifische Form sozialen Handelns zu verstehen. Eine solche Perspektive ist einerseits sinnvoll, andererseits unbefriedigend. Sinnvoll ist sie, weil sich gar nicht abstreiten lässt, dass Erziehung eine soziale Praxis ist (ebenso wie Religion, aber auch Medizin, Rechtsprechung oder das Kochen einer Gulaschsuppe). Es gibt daher keinen Grund, sich von einer Erziehungssoziologie zu verabschieden. Wie jede andere Disziplin auch ist die Erziehungswissenschaft auf die Rezeption von Forschungsergebnissen ihrer Nachbardisziplinen angewiesen, v. a. um die eigenen epistemologischen Grenzen zu überwinden. Unbefriedigend ist eine Quasi-Gleichsetzung (mit der Spezialisierung auf eine bestimmte Art sozialen Handelns), weil Erziehungswissenschaft etwas anderes sein müsste als Erziehungssoziologie oder konsequenterweise als Spezialdisziplin in sie aufzugehen hätte. So argumentiert auch Ronald Hitzler:

,[...] empirische Erziehungsforschung ist nichts anderes als eine thematische Spezialisierung unter vielen anderen der empirischen Sozialforschung schlechthin. Es mag zwar plausible historische, politische und pekuniäre Gründe dafür geben, dass die empirische Erziehungsforschung in pädagogischen und nicht in sozialwissenschaftlichen Instituten und Fakultäten angesiedelt ist. Von der ,Logik ' der Sache her aber gehört sie meines Erachtens nicht in die Pädagogik.“ (Hitzler 2008, S. 153)

Wenn wir dies anhand von Peter Vogels Beispiel auf andere Wissenschaften übertragen und weiterdenken, wird die Problematik daran schnell sichtbar: Man stelle sich vor, die Rechtswissenschaft würde sich mit dem (nicht von der Hand zuweisendem) Argument, dass die Rechtsprechung (auch) eine soziale Praxis sei, in eine reine Rechtssoziologie verwandeln. ${ }^{2}$ Für Rechtswissenschaftler und Rechtswissenschaftlerinnen ist dies wohl undenkbar, Erziehungswissenschaftler und Erziehungswissenschaftlerinnen hingegen scheinen damit allzu oft kein Problem zu haben.

\section{Pädagogisierung des Gegenstandes}

Indes zeichnen sich immer mehr Ansätze in der Erziehungswissenschaft ab, die versuchen, über die Integration von erziehungswissenschaftlichen Theorien in die qualitative Bildungsforschung den Gegenstand zu pädagogisieren. Dies betrifft insbesondere die bildungstheoretische Biographieforschung (vgl.

2 Gleiches ließe sich für Geschichtswissenschaftler, Ökonomen oder Literaturwissenschaftler etc. postulieren. Ohne Frage, auch die Geschichte ist eine soziale Praxis, genauso wie wirtschaftliches Handeln oder Literatur. Andererseits zeichnen sich die wissenschaftlichen Disziplinen durch eine besondere kognitive Spezialisierung aus, die sich auch institutionell sedimentiert hat. Die Disziplinen kultivieren damit einen spezifischen Blick auf die Welt (vgl. Stichweh 2013, S. 17). 
Marotzki 1999), aber auch zunehmend die qualitative Unterrichtsforschung (vgl. Meseth 2010). Sobald bildungsphilosophische und pädagogische Theorien in die qualitative Bildungsforschung integriert werden, zeichnen sich jedoch auch Probleme ab, die häufig insbesondere die implizite oder explizite Normativität der pädagogischen Begriffe betreffen.

Das Hauptmotiv, Pädagogik in Sozialwissenschaft transformieren zu wollen, liegt gerade darin, sich des normativen Ballasts der pädagogischen Erziehungs- und Bildungsphilosophie zu entledigen. Im Kontrast dazu wird in manchen Ansätzen das Normative der Pädagogik nicht als Ballast, sondern als das konstitutive Element einer genuin pädagogischen Forschung verstanden. Diese Autoren argumentieren genau gegenteilig. Qualitative Forschung wird als Ausgangspunkt einer normativen Kritik gesehen, quasi mit einem ,pädagogischen Blick“" (vgl. z.B. Gruschka 2013, S. 44) verbunden. Dies korrespondiert mit dem außerwissenschaftlichen Anspruch, der darin besteht, Erziehungsverhältnisse zu verbessern, was - da es sich um eine Form der Handlungswissenschaft handle - nicht negiert werden dürfe (vgl. Krummheuer/Najouk 1999, S. 14). Genau darin wird die Aufgabe pädagogischer Forschung gesehen. Für qualitative Verfahren ist dies vor allem deshalb problematisch, weil es in vielen Fällen dazu führt, statt einer offenen und rekonstruktiven eine evaluierende und bewertende Perspektive einzunehmen (ausführlich wird dies in Beier/Wyßuwa 2016 diskutiert; vgl. auch Meseth 2011).

Dies betrifft m. E. auch die bildungstheoretisch orientierte Biographieforschung, in der hinterfragt wird, inwieweit dem Bildungsbegriff selbst nicht eine implizite Normativität unterliegt und, inwiefern normative Implikationen des Bildungsbegriffs im Forschungsprogramm der bildungstheoretisch orientierten Biographieforschung deutlicher zu berücksichtigen“" (Fuchs 2014, S. 127) sind. Ziel einer normativ gehaltvollen bildungstheoretischen Biographieforschung ist es zwar dann nicht, Verbesserungsvorschläge oder Handlungsempfehlungen zu geben, aber doch die normativen Bildungstheorien empirisch „zu füllen“.

Das Anlegen eines normativen Maßstabs hat zur Konsequenz, dass letztlich darüber geurteilt wird, ob Bildungsprozesse stattfinden oder nicht. In der Unterrichtsforschung wird beispielsweise die Handlung des Lehrers oder der Lehrerin kritisiert und in der Professionsforschung professionelles von unprofessionellem Handeln geschieden. So kritisiert Gruschka beispielsweise das Verschwinden der Sache an sich zugunsten von formalen Präsentationstechniken im Unterricht oder beklagt eine zunehmende „,ürgerliche Kälte“ in der Lehrpraxis. Thorsten Fuchs' Biographieanalysen hinterfragen, wie viel Bildungsprozesse tatsächlich hinter einer vordergründigen Aufstiegsbiographie stecken. Außerdem stellt er wie andere Autoren (z.B. Koller 1999) zur Disposition, ob tatsächlich alle Wandlungsprozesse als Bildungsprozesse verstanden werden können, wie dies Winfried Marotzki (1990) vorgeschlagen hat, oder ob für ein angemessenes Bildungsverständnis nicht auch normative Aspekte eine Rolle 
spielen müssen. Fraglich bleibt, inwieweit solche Analysen tatsächlich einen Beitrag zu einer erziehungswissenschaftlichen Theoriebildung leisten können.

Normative Aussagen haben für eine auf Falsifikation angelegte empirische Forschung die unangenehme Eigenschaft, dass sie schlechterdings nicht widerlegbar sind. ${ }^{3}$ Normative Aussagen sind, wie nicht nur Niklas Luhmann (Luhmann 1969, S. 39) konstatierte, „lernresistent“. Der normative Maßstab lässt sich folglich durch empirische Forschung nicht widerlegen. Normative Ansätze tendieren daher dazu, Theorien weder empirisch hervorzubringen noch eine solche ernsthaft zu irritieren. Die gesetzte und nicht-widerlegbare Norm wird am Fall illustriert und ,gefüllt". Eine solche Forschung gerät in Gefahr, allein (mehr oder weniger wohlbegründete) Kritik zu üben, jedoch keine erfahrungswissenschaftliche Theoriearbeit zu leisten. ${ }^{4}$ Auch das Technologiedefizitproblem (vgl. Luhmann/Schorr 1982) wird eine solche kritische Forschung kaum überwinden, weil es fragwürdig bleibt, wie aus einer als schlecht identifizierten Praxis logisch korrekt darauf geschlossen werden kann, wie etwas besser gemacht werden müsste. Nur weil ich (scheinbar) weiß, dass sich jemand pädagogisch unangemessen verhält, weiß ich noch nicht, welche Konsequenzen (und welche unintendierten Nebenfolgen) vermeintlich ,pädagogisch wohlgeformtes“ Verhalten gehabt hätte (vgl. dazu Breidenstein 2012, S. 95). Dort, wo das Pädagogische an der qualitativen Forschung darauf gerichtet wird, bildungsphilosophische Kritik zu üben und hypothetische Handlungsempfehlungen zu generieren, wird sie sich kaum überzeugend als eigene sozialwissenschaftlich orientierte Forschungsdisziplin durchsetzen können. Das Pädagogische in die qualitative Forschung über

3 In der Tat betrifft dies nicht allein normative Aussagen, sondern auch theoretische Grundlagentheorien, die empirischen Analysen - werden sie nicht positivistisch verkürzt - immer zugrunde liegen. Über die Falsifikationsmöglichkeiten verschiedener Theorieelemente in der qualitativen Forschung informiert auch der Aufsatz von Gesa Lindemann (2015). In diesem Sinne ist das pauschale Argument, dass normative Aussagen nicht mit empirischer Forschung zu vereinbaren sei, nicht vorbehaltlos gültig. Die zu diskutierende Frage wäre vielmehr, inwieweit normative Theorien als Gegenstandstheorien fungieren können.

4 Dies kann offenbar nur dann geschehen, wenn eine Norm zu einem analytischen Begriff transformiert wird. Zum Beispiel, indem der Bildungsbegriff eine spezifische Wendung erhält, dessen Entstehungsbedingungen etc. dann analysiert werden (Was ermöglicht Bildung?). Dies ist allerdings weniger normativ - wie häufig behauptet wird - als vielmehr eine Operationalisierungsentscheidung, die eine notwendige Dezision im Forschungsprozess darstellt und zum Kerngeschäft empirischer Arbeit zählt (vgl. Vogel 2016: 231). Ein Begriff lässt sich weder normativ als richtig begründen noch empirisch als wahr erweisen. Anders verhält es sich, wenn Situationen, Biographien, etc. interpretiert werden und dann Handlungen (etc.) bewertet und vor dem Hintergrund einer „besseren Praxis“ analysiert werden. Dann wird eine empirische Analyse normativ. Inwieweit der dieser Bewertungspraxis zugrunde liegende normative Maßstab jedoch durch Empirie irritierbar und nicht Teil einer kontingenten Praxis ist, bleibt die darauf bezogene methodologische Problemstellung, die es zu lösen gilt. Dieses Problem wird im Ansatz zumindest von Andreas Gruschka angegangen (vgl. Gruschka 2011). 
Kritik und Normativität zurückzuholen und pädagogisch angemessene Handlungsalternativen aus Interview- oder Beobachtungsprotokollen abzuleiten, ist daher m. E. ein aussichtsloser Versuch, soziologische Auswertungsverfahren mit pädagogischer Relevanz zu versehen. In der Tat wird dies nicht zuletzt von Soziologen auch immer wieder kritisiert und führt zu einer Abseitsstellung im Feld der Bildungsforschung.

\section{Gibt es einen Ausweg? Der pädagogische Blick als qualitative Forschungsmethodologie - ein Desiderat einer erziehungswissenschaftlichen Bildungsforschung}

Ohne Zweifel gibt es in beiden - eher idealtypisch dargestellten - Positionen immer wieder reflexive Bezugnahmen einerseits zu den pädagogischen Ansprüchen, die offenbar nicht gänzlich ohne Normativität zu denken sind, und andererseits zu den methodologischen Grundlagen einer Sozialwissenschaft, die diese zu verbieten scheinen. Selten wird dabei jedoch die jeweilig skizzierte Grundposition verlassen. Daher ging es mir weniger um eine differenzierte Darstellung als vielmehr um die Illustration eines Grundproblems: Dass die Erziehungswissenschaft innerhalb der Bildungsforschung eine untergeordnete Rolle spielt - so die These - liegt vor allem an diesem wenig geklärten Verhältnis. Einen ernsthaften Beitrag zur interdisziplinären Bildungsforschung kann die Erziehungswissenschaft aus meiner Sicht nur dann leisten, wenn sie in der Lage ist, den sozialwissenschaftlichen Gütekriterien gerecht zu werden und mit einem anschlussfähigen, erfahrungswissenschaftlichen und deskriptiven Theoriebegriff operiert, zugleich aber eine autonome und erkenntnisfördernde Perspektive und d.h. eine eigenständig pädagogische (und möglicherweise normative) Gegenstandskonstitution begründen kann. Diese Gegenstandskonstitution kann meines Erachtens nicht als pädagogische Heuristik funktionieren, die ad hoc als Interpretationsleistung auf qualitative Daten aufgelegt wird. Vielmehr müsste eine pädagogische Theorie als Grundlagentheorie einer qualitativen Analysetechnik entwickelt werden, die begründet, warum etwas als pädagogisches Datum erscheint. In diesem Sinne fehlt es noch an einer dezidiert pädagogisch begründeten qualitativen Methodik, auch wenn erste Ansätze dazu bereits insbesondere in der Biographieforschung erkennbar sind. Dies macht darauf aufmerksam, dass auch die Erziehungswissenschaft keine ihr völlig ,eigenständige Methodik“ benötigt, die sich von sozialwissenschaftlichen Gütekriterien verabschiedet. Vielmehr geht es darum, anstatt soziologische Verfahren zu pädagogisieren, eigenständige qualitative Analysezugänge zu finden, die stärker auf pädagogischen Gegenstandskonstitutionen beruhen. Im Zentrum muss also die Frage stehen, wie Daten als erziehungswissenschaftlich relevante Daten konstituiert 
werden. Nur eine solche eigenständige Perspektive könnte das interdisziplinäre Feld der Bildungsforschung ernsthaft bereichern.

Frank Beier, Dr. phil., ist wissenschaftlicher Mitarbeiter an der TU Dresden.

\section{Literatur}

Asbrand, Barbara/Martens, Matthias/Petersen, Dorthe (2013): Die Rolle der Dinge in schulischen Lehr-Lernprozessen. In: Nohl, A.-M./Wulf, C. (Hrsg.): Mensch und Ding. Die Materialität pädagogischer Prozesse (= Zeitschrift für Erziehungswissenschaft. Sonderheft 25), S. 171-188. https://doi.org/10.1007/s11618-013-0413-1.

Beier, Frank/Wyßuwa, Franziska (2016): Präskriptive und reflexive Erkenntnisinteressen in der qualitativen Kurs- und Unterrichtsforschung. In: Miethe, I./Kreitz, R./Tervooren, A. (Hrsg.): Theorien in der qualitativen Bildungsforschung - Qualitative Bildungsforschung als Theoriegenerierung. Opladen: Verlag Barbara Budrich, S. 84-104.

Berndt, Constanze/Häcker, Thomas/Leonhard, Tobias (Hrsg.) (2017): Reflexive Lehrerbildung revisited. Traditionen - Zugänge - Perspektiven. Bad Heilbrunn: Klinkhardt.

Breidenstein, Georg (2012): Zeugnisnotenbesprechung. Zur Analyse der Praxis schulischer Leistungsbewertung. Opladen: Verlag Barbara Budrich.

Breidenstein, Georg/Menzel, Christin/Rademacher, Sandra (2013): Legitime und illegitime Differenzen im individualisierten Unterricht. Beobachtungen aus einer Montessori-Schule. In: Budde, J. (Hrsg.): Unscharfe Einsätze. (Re-)Produktion von Heterogenität im schulischen Feld (= Studien zur Schul- und Bildungsforschung 42). Wiesbaden: Springer VS, S. 153-167. https://doi.org/10.1007/978-3-531-19039-6_7.

Brezinka, Wolfgang (1971): Von der Pädagogik zur Erziehungswissenschaft. Eine Einführung in die Metatheorie der Erziehung. Weinheim: Beltz.

Dausien, Bettina (2007): Reflexivität, Vertrauen, Professionalität. Was Studierende in einer gemeinsamen Praxis qualitativer Forschung lernen können. Diskussionsbeitrag zur FQS-Debatte „Lehren und Lernen der Methoden qualitativer Sozialforschung". In: Forum Qualitative Sozialforschung 8,1, http://nbn-resolving.de/urn:nbn:de:0114-fqs0701D4Da3 [Zugriff: 13. März 2018]. http://dx.doi.org/10.17169/fqs-8.1.220.

Dinkelaker, Jörg/Meseth, Wolfgang/Neumann, Sascha/Rabenstein, Kerstin (2016): Die Erziehungswissenschaft, ihr Gegenstand und ihre Empirie. Sondierungen im Spannungsfeld von traditionellen Kontroversen und reflexiver Empirisierung. In: Meseth, W. et al. (Hrsg.): Empirie des Pädagogischen und Empirie der Erziehungswissenschaft. Beobachtungen erziehungswissenschaftlicher Forschung. Bad Heilbrunn: Klinkhardt, S. 13-30. 
Fuchs, Thorsten (2014): „Das war das Bedeutendste daran, dass ich mich so verändert habe“. Mit Ehrgeiz und Ansporn über Umwege zum Ziel - Der ,Bildungsweg' Hakans. Oder: Ist jede Transformation des Welt- und Selbstverhältnisses sogleich bildungsbedeutsam? In: Koller, H.-C./Wulftange, G. (Hrsg.): Lebensgeschichte als Bildungsprozess? Perspektiven bildungstheoretischer Biographieforschung. Bielefeld: transcript, S. 127-151.

Gruschka, Andreas (2011): Der empirische Blick auf das Unterrichten als pädagogischer Prozess. In: Proske, M./Meseth, W. et al. (Hrsg.): Unterrichtstheorien in Forschung und Lehre. Bad Heilbrunn: Klinkhardt, S. 130-145.

Gruschka, Andreas (2013): Unterrichten - eine pädagogische Theorie auf empirischer Basis. Opladen u. a.: Verlag Barbara Budrich.

Gräsel, Cornelia (2015): Was ist empirische Bildungsforschung? In: Reinders, H./Ditton, H./Gräsel, C./Gniewosz, B. (Hrsg.): Empirische Bildungsforschung. Strukturen und Methoden. Wiesbaden: Springer VS, S. 15-29. https://doi.org/10.1007/978-3-531-19992-4_1.

Helsper, Werner (2001): Praxis und Reflexion. Die Notwendigkeit einer „,doppelten Professionalisierung“ des Lehrers. In: Journal für Lehrerinnen- und Lehrerbildung, 1 (3), S. 7-15.

Hummrich, Merle/Hebenstreit, Astrid (2017): Disziplinierende Ordnungen Potenziale und Herausforderungen international vergleichender Ansätze für die erziehungswissenschaftliche Forschung. Vortrag auf der Tagung der DGfE-Kommission Qualitative Biographie- und Bildungsforschung (QBBF), 22.09.2017, Chemnitz. https://www.researchgate.net/publicati on/320131676 [Zugriff: 13. März 2018].

Hitzler, Ronald (2008): Grenze der disziplinären „Ökumene“. Zur fundamentalen Differenz von Jugendsoziologie und Pädagogik. In: Soziologie 37, 2, S. 145-154.

Koller, Hans-Christoph (1999): Bildung und Widerstreit. Zur Struktur biographischer Bildungsprozesse in der (Post-)Moderne. München: Fink.

Knoblauch, Hubert (2015): Sinn und Subjektivität in der qualitativen Forschung. In: Kalthoff, H./Hirschauer, S./Lindemann, G. (Hrsg.): Theoretische Empirie. Zur Relevanz qualitativer Forschung. Frankfurt am Main: Suhrkamp, S. 210-233.

Krummheuer, Götz/Najouk, Natalie (1999): Grundlagen und Beispiele Interpretative Unterrichtsforschung. Opladen: Leske + Budrich. https://doi.org/ 10.1007/978-3-322-95191-5.

Lindemann, Gesa (2015). Theoriekonstruktion und empirische Forschung. In: Kalthoff, H./Hirschauer, S./Lindemann, G. (Hrsg.): Theoretische Empirie. Zur Relevanz qualitativer Forschung. Frankfurt am Main: Suhrkamp, S. 107-128.

Luhmann, Niklas (1969/2008): Normen in soziologischer Perspektive. In: Luhmann, N.: Die Moral der Gesellschaft, hrsg. v. D. Horster. Frankfurt am Main: Suhrkamp, S. 25-55. 
Luhmann, Niklas/Schorr, Karl Eberhard (1982): Das Technologiedefizit der Erziehung und die Pädagogik. In: Luhmann, N./Schorr, K. E. (Hrsg.): Zwischen Technologie und Selbstreferenz. Frankfurt am Main: Suhrkamp, S. 11-41.

Marotzki, Winfried (1990): Entwurf einer strukturalen Bildungstheorie. Biographietheoretische Auslegung von Bildungsprozessen in hochkomplexen Gesellschaften. Weinheim: Deutscher Studien Verlag.

Marotzki, Winfried (1999): Erziehungswissenschaftliche Biographieforschung. Methodologie - Tradition - Programmatik. In: Zeitschrift für Erziehungswissenschaft 2, 3, S. 325-341.

Meseth, Wolfgang (2010): Aufbruch zu neuen Ufern empirischer Bildungsforschung. Disziplinäre Verortung, Fragestellung und Forschungsprogramm der Netzwerkinitiative „Methodologien einer Empirie pädagogischer Ordnungen“. In: Neumann, S. (Hrsg.): Beobachtungen des Pädagogischen. Programm - Methodologie - Empirie. Luxemburg: Universität Luxemburg, S. 15-26.

Meseth, Wolfgang (2011): Erziehungswissenschaftliche Forschung zwischen Zweck- und Wertrationalität. Überlegungen zum Normativitätsproblem der empirischen Unterrichtsforschung. In: Zeitschrift für Soziologie der Erziehung und Sozialisation 31, 1, S. 12-27.

Meseth, Wolfgang (2008): Schulisches und außerschulisches Lernen im Vergleich. Eine empirische Untersuchung über die Vermittlung der Geschichte des Nationalsozialismus im Unterricht, in außerschulischen Bildungseinrichtungen und in Gedenkstätten. In: kursiv. Journal für politische Bildung 12, 1, S. 74-83.

Reichertz, Jo (2014): Empirische Sozialforschung und soziologische Theorie. In: Baur, N./Blasius, J. (Hrsg.): Handbuch Methoden der empirischen Sozialforschung. Wiesbaden: Springer VS, S. 65-80. https://doi.org/10.1 007/978-3-531-18939-0_2.

Stichweh, Rudolf (2013): Wissenschaft, Universität, Professionen. Soziologische Analysen. Bielefeld: transcript.

Vogel, Peter (2016), Wie pädagogisch muss, kann, darf erziehungswissenschaftlich-empirische Forschung sein? In: Meseth, W. et al. (Hrsg.): Empirie des Pädagogischen und Empirie der Erziehungswissenschaft. Beobachtungen erziehungswissenschaftlicher Forschung. Bad Heilbrunn: Klinkhardt, S. 225-234.

Wyßuwa, Franziska/Beier, Frank (2013): Beispielerzählungen und Szenarioentwicklung in der Weiterbildung als Veranschaulichungen von Wissen und Relevanzen. In: Birkner, K./Ehmer, O. (Hrsg.): Veranschaulichungsverfahren im Gespräch. Mannheim: Verlag für Gesprächsforschung.

Zedler, Peter/Döbert, Hans (2010): Erziehungswissenschaftliche Bildungsforschung. In: Tippelt, R./Schmidt, B. (Hrsg.): Handbuch Bildungsforschung. Wiesbaden: VS Verlag, S. 23-45. https://doi.org/10.1007/978-3531-92015-3_2. 